\title{
MONTEIRO LOBATO: UM BRASILEIRO EM TRÂNSITO ${ }^{1}$
}

\author{
Marisa Lajolo* \\ Universidade Estadual de Campinas - UNICAMP \\ Universidade Mackenzie - São Paulo
}

A Nize Therezinha Martins Antunes

In Memoriam

Entre 7 de junho de 1927 e 19 de março de 1931 o escritor Monteiro Lobato (José Bento Monteiro Lobato, 1882-1948) viveu em New York, onde desempenhava a função de Adido Comercial à representação diplomática brasileira. Acompanhando sua farta correspondência, ficamos sabendo um pouco de sua vida nova-iorquina: seu primeiro endereço foi Jackson Heights, de onde se mudou para a Broadway. Era dono de um automóvel, porém usuário entusiasta do metrô, pelo qual chegava ao emprego, no prédio do Consulado Brasileiro, em Battery Place. Além de cidadão nova-iorquino, Monteiro Lobato foi personagem de sua imprensa.

Em 17 de agosto de 1927, seu nome aparece no New York Times. Trata-se, aparentemente, de um anúncio indireto da firma que lhe alugou o apartamento: por um lado, o destaque que o anúncio confere ao cargo de Lobato sugere que alugar um imóvel para um membro do corpo consular era uma recomendação para a imobiliária. Por outro, ao sublinhar a elegância da localização do imóvel e o status de outros

\begin{tabular}{|l|l|l|l|l|}
\hline Ilha do Desterro & Florianópolis & no 57 & p. 037-057 & jul./dez. 2009 \\
\hline
\end{tabular}


inquilinos, o anúncio sugere o nível de vida a que Lobato aspira enquanto nos Estados Unidos, uma vez que aluga uma residência em um conjunto que serve de grife para a imobiliária

\section{APARTMENT LEASES}

Brazilian Attaché Rents Suite at Jackson Heights

A garden apartment at Jackson Heights has been leased by J.B.Monteiro Lobato, Commercial Attaché of the Brazilian Embassy, through the Queensboro Corporation. Other recent lessees of garden apartments at Jackson Heights include John P.Allen and Martin Linsley. ${ }^{2}$

Na época, o Ministro de Estado de Relações Exteriores do Brasil era Octavio Mangabeira, o Embaixador do Brasil em Washington Sylvino Gurgel do Amaral, e o Cônsul Geral do Brasil em Nova York a quem Monteiro Lobato era diretamente subordinado-Sebastião Sampaio. Esse último já cruzara caminhos com seu Adido Comercial. Tinham sido colegas na Faculdade de Direito de São Paulo e o futuro Cônsul protagonizara uma situação um tantinho desqualificada, relatada em 1908 por Monteiro Lobato a Godofredo Rangel, seu excompanheiro de escola e correspondente de vida toda:

\section{S.Paulo, 25,7,1908}

... íamos descendo a Rua Direita, rumo ao Viaduto, quando aparece o Sebastião Sampaio e adere. E como viu que o Ricardo recitava , mete a mão no bolso e diz, sacando o papel: "Eu também tenho aqui uns versos que vou ler...". Ninguém, pronunciou uma palavra. Não houve comentário nem combinação nenhuma. No maior una voce mudo que jamais vi, todos nos pusemos a correr e só paramos para lá do Viaduto, no começo da rua Itapetininga. Só então nos 
voltamos. A garoa leve dava para distinguir o vulto do Sampaio no princípio do Viaduto, com uma coisa branca na mão. (Lobato, A barca 1:134-35)

Deste Sebastião Sampaio que fazia versos em 1908 nada parece ter restado ao chefe de Monteiro Lobato em Nova York nos anos 20. Diplomata de carreira, ele é signatário de grande parte da correspondência relativa ao período novaiorquino de Lobato, correspondência bastante ortodoxa, exceto em poucas ocasiões como quando, por exemplo, desmente a informação de seu falecimento:

A "United Press" comunica que foi noticiado, aí, ter eu sido assassinado aqui, ontem. Ouso pedir a Vossa Excelência telegrafar Madame Sebastião Sampaio, residência Waldemar Mercadante, Limeira, Estado de São Paulo, que estou de perfeita saúde. $^{3}$

Igualmente pouco ortodoxo é o registro mais antigo da presença de Monteiro Lobato na diplomacia brasileira. Em 26-27 de maio de 1927, um telegrama da embaixada brasileira em Washington dirigido ao Ministério das Relações Exteriores indagava da veracidade da nomeação do escritor para o posto de Adido Comercial: "Monteiro Lobato telegrafou dizendo ter sido nomeado Adido Comercial. Peço confirmação oficial, indicando seu nome inteiro e se vem acompanhado de família. AMARAL". ${ }^{4}$

No dia seguinte (28.05.1927), um discreto telegrama, na linguagem entrecortada do gênero, confirmava a nomeação: "José Bento Monteiro Lobato nomeado comissão adido portaria onze Abril durante impedimento efetivo expediente seguiu mala. Leva Senhora quatro filhos governanta". ${ }^{5}$ Mas se pouca ortodoxia na correspondência do corpo diplomático parece ser exceção, na de Monteiro Lobato era a regra. Sua presença nos quadros da diplomacia brasileira parece marcada, desde o início, por turbulências. Nem bem havia ele desembarcado e já 
o cônsul antevia problemas para sua acomodação nas dependências do Consulado:

Senhor Ministro de Estado ... V.Ex., no telegrama no. 51, determina que devo alojar o Sr. Adido Comercial. Este Consulado terá muita honra e o maior prazer em cumprir essa ordem de V. Ex. O Senhor Adido Comercial poderá escolher a sala onde colocar a sua mesa de trabalho. Julgo de meu dever, entretanto, ponderar que, sem uma sala especial, para o que serão necessárias mais dez ou doze libras mensais, o Senhor Adido ficará muito mal instalado. Se V. Ex. concedeu as duas saletas afim (sic) de que uma ficasse para uso exclusivo do Sr. Adido, devo dizer a V.Ex. que a falta de espaço no Consulado continuaráa mesma, cabendo ao Adido Comercial um cômodo escuro, pequeno, sem luz, útil apenas a este Consulado Geral, por ser um prolongamento de suas salas e por um preço que permitiu o aumento de verba de aluguel.

Talvez não seja impróprio tomar esta falta de espaço para Lobato, ou as tumultuadas formas pelas quais este espaço é conseguido como metáfora de suas tensas relações com o que a diplomacia representava do Estado brasileiro.

Também a figura do Cônsul Sebastião Sampaio que emerge da correspondência e da documentação relativa a Monteiro Lobato ganha um perfil instável, sugerindo um relacionamento pessoal e profissional cheio de altos e baixos. Em carta de 20 de junho de 1929, ao mesmo Godofredo Rangel, o adido comercial constrói uma imagem pouco lisonjeira de seu chefe. Atribui a Sebastião Sampaio manipulação de notícias que davam conta de imagens do Brasil nos Estados Unidos, como o falso sucesso da Miss Brasil ${ }^{6}$ daquele ano nos Estados Unidos:

Miss Brasil, coitadinha, passou absolutamente desapercebida aqui .... Foi com dificuldade que consegui saber o resultado 
desse concurso, onde a pobre menina foi desclassificada, não obtendo nenhum dos onze lugares. $\mathrm{O}$ fato é esse. $\mathrm{O}$ mais é Cônsul Sampaio e reporters. Mas pelos jornais hás de ter visto como este nada foi transformado em tremenda glorificação da beleza indígena. Manipulação pura ! ... Há dias assisti à germinação de outra festa. Esteve cá um figurão da medicina Brasília e o cônsul arranjou-lhe um telegrama para o Rio dizendo que ele fora ou ia ser homenageado em Chicago ou Filadélfia com um banquete de 400 mestres da ciência americana. (Lobato, $A$ barca 2: 317-18)

Se os solavancos de Monteiro Lobato com seu chefe talvez não surpreendam, surpreendem alguns silêncios lobatianos relativos a assuntos e iniciativas em curso na representação diplomática brasileira ao tempo em que ele foi adido comercial. Um desses assuntos é a revista Brazil. Cinco meses depois do desembarque de Lobato, o consulado de Nova York inicia a publicação da revista Brazil de longa duração e de cujo primeiro número o Museu do Itamaraty guarda um exemplar que se abre com uma espécie de carta de intenções :

\section{BRAZIL}

\section{A MAGAZINE PUBLISHED MONTHLY BY THE AMERICAN-BRAZILIAN ASSOCIATION}

New York City, November, 1927, n I

An Announcement

FOUNDED by the iniciative of the Hon. Sebastião Sampaio, Consul General of Brazil, both the American Brazilian Association and its magazine "BRAZIL" are already real facts, and most useful instruments to increase social, cultural and commercial relations between the two greatest Republics of 
this continent. The example of the New York friends of Brazil, who so well supported this project, must be followed by all the other friends the Southern nation has throughout this country, and by all Brazilians interested in closer relations with the United States.

We are receiving applications for our membership lists: Corporate Members, Individual Members and Associate Members. Please see the next page for details.

Tuesday, November $15^{\text {th }}$, BRAZILIAN DAY, we will celebrate our formal inauguration with a luncheon at the Bankers Club at 12:45PM. We hope all friends of Brazil in New York will attend the luncheon on that day.

\section{THE AMERICAM BRAZILIAN ASSOCIATION}

Thirty cents a single copy - Year's subscription, Three Dollars (in advance).

For advertisements, subscription, editorial and all other matters-Address: 17 State Street, Room 1015, New York City.

A menção a "the Hon. Sebastião Sampaio, Consul General of Brazil", a caracterização da revista como vinculada a uma "American Brazilian Association", a alusão a outra provável associação, "a New York Friends of Brazil", o objetivo declarado de "increase social, cultural and commercial relations", ao lado da generosa identificação entre Brasil e Estados Unidos como "the two greatest Republics of this continent" delineiam um perfil gramscianamente orgânico para a revista, perfil que talvez se reforce pela notícia de brasileiros em missão oficial nos Estados Unidos, como por exemplo, Anísio Teixeira, de quem, aliás, Monteiro Lobato vai tornar-se amigo:

Public Instruction in Bahia. The distinguished Governor of the State of Bahia, Dr. Goes Calmon, has sent to the United State Dr. Anisio Teixeira, his Director of Education, to buy material and to study new methods for the Public Schools of 
Bahia. Dr. Teixeira returned to Bahia a few days ago, after fulfilling his commission here.

Também os anúncios veiculados pela revista constroem uma imagem eufórica do Brasil, ao aproximar, por exemplo, café e eletricidade:

\section{COFFEE AND ELECTRICITY}

The relationship between this delightful beverage and electricity is closer than the unthinking realize. From the consumption end alone, one powerful group of electrical companies sold 11,352 electric percolators during the calendar year of 1926. Estimating this number as one-tenth the total sales for the year throughout the United States gives us a picture of 100,000 newly installed electric coffee percolators singing their pleasant morning song at as many American Breakfast tables and filling the surrounding air with the delightful fragrance of this unmatchable beverage. From the production end, we find a group of electric companies stretching wires throughout the northwestern section of the state of São Paulo, Brazil, the greatest coffee growing region in the world. Here, in an area covering nearly 40.000 square miles, 462.000 .000 coffee trees blossom in the mild and delightful climate of the Brazilian uplands, producing with the aid of electricity, the berry so indispensable to the American breakfast. Empreza Força e Luz de Ribeirão Preto

Uma corda nostálgica de Monteiro Lobato poderia sensibilizar-se pela menção de Ribeirão Preto, cidade do interior paulista que morou por algum tempo no coração do escritor, que com ela sonhava ao tempo em que buscava nomeação para alguma promotoria no interior paulista. Para ele, Ribeirão Preto era o reverso das cidades mortas. Como registra o anúncio, Ribeirão Preto era "the greatest coffee growing region in the world." Apesar de mencionado na publicação que nomeia todo o staff 
do consulado, Monteiro Lobato abstém-se de comentá-la. Talvez se possa atribuir ao caráter institucional da publicação a completa omissão da revista nos registros de Monteiro Lobato, omissão que se torna estranha por Monteiro Lobato já ser, quando chega aos Estados Unidos, um escritor de circulação muito ampla no Brasil e traduzido para Espanhol, Francês e Inglês.

Documentos da Cia. Editora Nacional ${ }^{7}$ indicam até 1925 a venda de 223.500 (duzentos e vinte e três mil e quinhentos) exemplares de suas obras e mais 28.000 (vinte e oito mil) no ano seguinte. Desde 1921 vários contos seus haviam sido publicados na Argentina, e alguns na França. Em 16.05.1921, Alma Negra (tradução de Negrinha) é publicado em A novela semanal. Em 1923, El comprador de haciendas (tradução de Benjamin de Garay de $O$ comprador de fazendas) integra uma coletânea de contos publicada na Argentina. Em abril de 1924, Barba Azul (tradução por Sanchez Saez de Barba Azul )é publicada na revista Argentina Haciendas. Em setembro de 1924, seu conto "A vingança da peroba" é publicado em francês (tradução de G. Le Gentil) no número 33 da Revue de l'Amérique Latine.

Em setembro de 1924, Isaac Goldberg (1887-1938) lança, como número 733 da coleção Little Blue Books (editado por Haldeman Julius ${ }^{8}$ ) Brazilian Short Stories, livro que se compõe de três contos de Monteiro Lobato: "Modern Torture," "The Penitent Wag" e "The Plantation Buyer". Estréia modesta: 61 páginas de um livrinho de $9.0 \mathrm{~cm}$ por $12.50 \mathrm{~cm}$. Originalmente incluídos em Urupês ${ }^{9}$, cada um destes contos, foi traduzido para o inglês por uma até agora não identificada "woman friend of Lobato resident in Brazil," conforme indica a introdução do pequeno volume, assinada por Goldberg.

Podemos, assim, dizer que, em papel e tinta, Monteiro Lobato chegou aos Estados Unidos três anos antes de sua chegada em carne e osso, pelo navio American Legion, a New York. Mas o Brasil que protagozinava este contos lobatianos era bem o avesso da imagem de Brasil que construía a diplomacia brasileira. Além da publicação da revista Brazil, outras iniciativas diplomáticas contemporâneas da 
experiência nova-iorquina de Monteiro Lobato traduzem a vontade de fortalecimento da imagem do Brasil no contexto norte-americano. Parecem trabalhar neste sentido, tanto a proposta de uso da língua portuguesa na União das Repúblicas Americanas, quanto a recusa em endossar atitudes anti-norte-americanas:

Senhor Embaixador, Tenho a honra de remeter a Vossa Excelência a inclusa copia de uma informação da Secção dos Negócios Políticos e Diplomáticos da América sobre o uso da língua portuguesa na União das Republicas Americanas. 2. Rogo a Vossa Excelência a bondade de examinar esse assunto, sobre o qual estimaria conhecer o seu parecer. Aproveito este ensejo para reiterar a Vossa Excelência os protestos da minha alta estima e mais distinta consideração. Octavio Mangabeira ${ }^{10}$

Senhor Embaixador,

Pelo Despacho confidencial PA/13 de julho último, dei conhecimento a Vossa Excelência da noticia aqui recebida da nossa Embaixada em Santiago, segundo a qual o México capitaneava, à frente dos países de América Central, um movimento contra os Estados Unidos da América na próxima Conferência Internacional Americana. Essa noticia teve-a o Embaixador Roças do Sub-Secretario das Relações Exteriores do Chile.

2. Acusando o recebimento daquele Despacho, disse-me Vossa Excelência, em substancia, no seu Officio Confidencial número 274, de 28 de agosto, que nada via aí pudesse corroborar os vaticínios pessimistas correntes em Santiago; $\mathrm{e}$ que os Estados Unidos da América, embora merecedores da oposição que lhes moviam certas correntes centro-americanas, acreditavam, contudo, que o prestígio de que goza seu Governo, e a sensatez das delegações. ${ }^{11}$ 
Como a revista Brazil, também essas questões passam completamente em branco na epistolografia lobatiana até hoje conhecida.

O caudaloso correspondente parece ter papel e tinta em primeiro lugar para seu profundo entusiasmo pelos Estados Unidos:

Cá estou, na maior cidade do mundo. É bem a maior . É tudo imenso, desconforme(?), acima de tudo quanto podemos imaginar. A nossa mentalidade brasileira, só vendo é que pode alcançar as proporções desta cidade infinita. Tudo diferente daí. A ordem, a disciplina, a facilidade (?) da vida, a riqueza do povo ... Quem não conhece esta civilização não está com o curso de vida completa. A Europa é zero diante disto. Todo mundo tem automóvel e não é Ford. O povo enriqueceu tanto que o Ford caiu. ... Imagine que o porteiro de nosso bloco possui um Cadilac! ... Vê-se o dinheiro rolar. Tudo cheio, hotéis, restaurantes, teatros, magazines. Em resumo, isto é tudo o contrário de nosso pobre Brasil, iludido com a besteira de ser Deus brasileiro. Deus é norteamericaníssimo! ${ }^{12}$

Mas este escritor, que nos arredores de sua chegada à América é todo louvores ao American way of life, vinte anos depois, registra em cartas uma formidável guinada ideológica:

S.Paulo, 2,1,1944.

Prezadíssimo Dr. Jorge Americano: Em mãos a sua de 28 de dezembro último. Nada de estranho há no meu pedido de retirada da união Cultural Brasil-Estados Unidos. Há apenas um pouco de lógica. Como verifiquei que os americanos fazem a maior das guerras ao fascismo na Europa e dão todo o apoio moral e material ao mesmo fascismo aqui, achei de bom conselho não contribuir para a união cultural entre os 
dois povos, de medo que o brasileiro acabe ainda mais semvergonha do que é. Como o prezado amigo vê, trata-se apenas dum bocadinho de lógica. Com a maior estima subscrevome Amo. E admor. A) Monteiro Lobato. (Lobato, Cartas 262)

São, assim, vários os aspectos e os tempos nos quais se desencontram o Monteiro Lobato morador de Nova York e os rumos da política externa brasileira. Mas há também encontros: mais tarde, já de regresso ao Brasil, o escritor mergulhará de corpo e alma em históricas, frustradas e polêmicas campanhas pelo petróleo e pelo ferro, personagens assíduos tanto da correspondência diplomática, quanto de dezenas de cartas que Monteiro Lobato escreve dos Estados Unidos até o final de sua experiência diplomática, final que parece ter vindo pelo decreto de 6 de dezembro de 1930, assinado pelo chefe do Governo Provisório, que dispensava vários funcionários do Itamaraty, Monteiro Lobato entre eles: "Por decreto de 9 do corrente passaram para o novo Ministério do Trabalho os Serviços Econômicos e os Adidos Comerciais, com as respectivas verbas e pessoal. EXTERIORES" ${ }^{13}$ Ferro e petróleo são ainda menção freqüente no livro América, que Monteiro Lobato lançou em 1932 com uma bela capa de J.U.Campos, e que teve tiragens expressivas: 6.000 exemplares em 1932, $3.000 \mathrm{em}$ 1934, $32000 \mathrm{em} \mathrm{1937.}{ }^{14}$

Se, como vimos, foi pelas mãos de Isaac Goldberg que se inaugurou um dos caminhos norte-americanos de Monteiro Lobato, também foi antes de viajar para New York que o escritor começou a incluir o cenário norte-americano em sua ficção. Entre 5 de julho e $1^{\circ}$ de outubro o jornal carioca A manhã (propriedade de Mário Rodrigues, pai do dramaturgo e jornalista Nélson Rodrigues), no qual Monteiro Lobato colaborava, começa a publicação, em folhetins, de $O$ presidente negro. Em dezembro, os folhetins foram reunidos em volume, dedicado a Artur Neiva e a Coelho Neto. O romance constitui uma ficção científica sociológica. $\mathrm{O}$ enredo transcorre no longínquo ano de 2228 e encena o resultado de uma eleição presidencial norte-americana, na qual um negro é eleito presidente . 
Ao mesmo tempo em que Monteiro Lobato, ainda no Brasil, escreve e publica este até hoje polêmico romance de ambientação norte americana, ele também palmilha outra trilha letrada para a América do Norte, através de uma série de artigos sobre Henry Ford, publicados no mesmo jornal A manhã. Esses artigos sobre Henry Ford foram passados para o inglês por Aubrey Stuart e, ao mesmo tempo, Lobato traduzia para português Minha vida e minha obra, de Henry Ford.

Deve ter chegado aos ouvidos do magnata norte americano a devoção do brasileiro: funcionários da Ford estavam à espera de Lobato e sua família e desembaraçaram a bagagem necessária para uma longa permanência de cinco pessoas, ficando as malas confortavelmente guardadas num dos armazéns da poderosa Ford Motor Company.

São, assim, vários os Monteiros e muitos os Lobatos que transitam pela América do Norte no final dos anos 20 e começo dos anos 30. E, da mesma forma, ao lado do Brasil que os documentos diplomáticos da época nos fazem descortinar, outros Brasis - no caso, Brazis- também transitam pela New York de Lobato.

Acompanhar algumas notícias sobre o Brasil publicadas pelo $\mathrm{NeW}$ York Times nos arredores da experiência nova-iorquina de Monteiro Lobato revela um Brasil tão em trânsito quanto o escritor.

Em 25 de maio de 1927, quando a família Lobato ultimava preparativos para rumar para os Estados Unidos, o sisudo New York Times noticiava - com a discrição com que até hoje informa seus leitores do que se passa fora das fronteiras norte-americanas - manifestação de rua contra o presidente Artur Bernardes, estropiando-lhe o nome:

Brazilians are hostile to Bernardes: Rio de Janeiro, Brazil, May 21 (AP) Former president Arturo (sic) Bernardes was the object of hostile demonstrations when he alighted from a train here last night. Afterwards he left in an automobile with a heavy escort of police. The crowds paraded through the principal streets of the city, shouting vivas for Brazil and "death to the tyrant". ${ }^{15}$ 
A notícia ilustra uma das perspectivas em que o Brasil comparece à imprensa norte-americana e ao imaginário de seus leitores. Nela se revela um jornal atento à cena brasileira, registrando manifestações políticas como curiosidades, a começar pelo equivocado nome do presidente Artur Bernardes mencionado como Arturo Bernardes. As "hostile demonstrations" são transformadas em arruaças, tornando-se o Rio de Janeiro numa espécie de território sem lei, onde "crowds paraded throught the streets of the city".

Na mesma época, alguns arquivos do Itamaraty ocupam-se do assunto, sugerindo presença forte da diplomacia brasileira junto a agências de notícias, na formatação de notícias relativas ao Brasil:

CONFIDENCIALE RESERVADO Senhor Ministro de Estado, No dia 26 ultimo, á tarde, o Sr. Presidente em exercício da United Press telefonou-me que acabava de receber do Rio um telegrama que me comunicou e que eu traduzo a seguir: "Uma grande força de policia formou cordão em redor do Palácio do Senado, para impedir violentas manifestações contra o Ex-presidente Arthur Bernardes, que tomou posse de sua cadeira de Senador. A policia teve de carregar contra $O$ povo para manter a ordem. A imprensa oposicionista noticia que algumas pessoas ficaram ligeiramente feridas. A demonstração terminou quando o Sr. Arthur Bernardes deixou o Senado. O Sr. Bernardes saiu para a Europa ás 11 horas da manhã, a bordo do paquete "Bagé", cuja tripulação foi substituída por outra, da confiança do Senador Bernardes. Uma nova demonstração hostil teve lugar no cais, mas não houve violências. OSenador Bernardes partiu para o Havre." Como V. Ex. vê, a noticia, apesar do que tem em si de muito desagradável, - se o fato é verdadeiro - está em termos que mostram a boa vontade habitual da United Press, não só não espalhando noticias alarmantes sobre o Brasil, como também pondo sempre em destaque a atitude prudente e correta do 
Governo Brasileiro.... Agradeci a gentileza do Sr. Presidente da United Press e pedi-lhe, confidencialmente, já que começara a enviar a noticia aos seus jornais, que não a comentasse, nem lhe desse destaque, no que fui imediatamente atendido. Comuniquei tudo, logo depois, pelo telefone, a Sua ex. o Sr. Embaixador em Washington. Anoticia em questão, felizmente, não causou nenhuma impressão pelo simples fato de passar desapercebida, publicada sem grandes títulos, e em poucas linhas, numa das numerosas paginas dos jornais americanos. Transcrevo, para exemplo, a noticia dada pelo "New York Times," pagina 25, da $2^{\mathrm{a}}$ edição de 27 de Maio: "Hoot Brazil ex-president. Hostile Demonstration Necessitates Senate Guard for Bernardes. Rio de Janeiro, May $26(A P)-A$ contingent of troops had to be sent to surround the Senate last night to guard against disorders by a hooting, whistling mob while former President Artur Bernardes was being sworn in as a member. He was elected to represent his State, Minas Gerais. Several days ago, when Senhor Bernardes alighted from a train in Rio de Janeiro, he was the object of a hostile demonstration, a large crowd shouting epithets and hissing him. The demonstrations are an outgrowth of indignation in some quarters over alleged cruelties during his Administration in a campaign to break up revolutionary plotting."

Tratando neste ofício de noticia transmitida pela United Press, julgo de meu dever transmitir uma informação de detalhe que V. Ex. talvez tenha interesse em conhecer.

Quando Chefe do Gabinete do Ministro, no Itamaraty, verifiquei que o atual representante da United Press, aí, o Sr. U. J. Keener, é pessoa digna de toda confiança, prudente, inteligente, com ordens do Presidente da United Press de seguir sempre, sobre noticias relativas ao governo brasileiro, as instruções que receber do Itamaraty. Como esse 
representante é, entretanto, pessoa muito tímida, talvez não tenha feito ainda relação com os auxiliares de V. EX., como fez antes comigo; estou certo, porem, de que atenderá ao primeiro chamado que receber e sempre consultará o Itamaraty, antes de mandar qualquer noticia que envolva certa importância política, - caso V. ex. julgue tal providencia conveniente.

Está claro que esta minha informação é de ordem geral, porque, no caso presente, o correspondente americano aí se limitou a telegrafar para Nova York, de maneira prudente, sem alarmes, - se o fato é verdadeiro, - uma noticia que, pela ética jornalística, não podia deixar de enviar, sob pena de ser, como se diz em imprensa, furado pelas outras agencias telegráficas. Aproveito a oportunidade, Senhor Ministro, para reiterar a V. Ex. os protestos de minha respeitosa consideração. Sebastião Sampaio (os parágrafos e itálicos são meus ML).

O texto acima é primoroso no que ensina relativamente a modos de controle da imagem do Brasil veiculada pela imprensa estrangeira. Já em seu início insinua-se o contacto rotineiro entre agências de notícias e a representação diplomática brasileira: "o Sr. Presidente em exercício da United Press telefonou-me ...". Segue-se à tradução do telegrama uma avaliação positiva da atitude da agência noticiosa : "boa vontade habitual da United Press" que sublinha o alinhamento entre a United Press e o governo brasileiro: "não só não espalhando noticias alarmantes sobre o Brasil, como também pondo sempre em destaque a atitude prudente e correta do Governo Brasileiro". Segue-se detalhamento de solicitações da representação brasileira, sugestões de maneiras de divulgação da notícia que atenuem seu eventual impacto: "pedi-lhe ... que não a comentasse, nem lhe desse destaque", o que parece ter sido plenamente conseguido, já que a notícia "não causou nenhuma impressão pelo simples fato de passar desapercebida, publicada sem grandes títulos, e em poucas linhas, numa das numerosas paginas dos jornais americanos". 
Trata-se, como se vê, de medidas que complementam e secundam a linha editorial da revista Brazil e que também manifestam a imaginação com a qual, segundo Monteiro Lobato, Sebastião Sampaio retoca matérias sobre a Miss Brasil ou sobre o médico visitante, montando o caleidoscópio de imagens do Brasil que se alternavam no contexto em que Monteiro Lobato viveu sua experiência norteamericana.

O exotismo com que a matéria sobre Artur Bernardes - ainda que editada por Sebastião Sampaio - parece tratar questões de política brasileira se acentua em outra notícia, de 28 de outubro de 1930 , vésperas de Getúlio tomar o poder e de demitir Monteiro Lobato.

\section{Zhetoolio Vahrgahs" Is Way To Say Brazilian Chief's Name}

With a new president about to take Office in Brazil, considerable interest was expressed yesterday in New York as to the correct pronunciation of his name. Inquiry at the Brazilian Consulate General brought the explanation that Getulio Vargas is pronounced Zhetoolio Vahrgahs with the accent on the first syllable of the President's surname. The soft " $g$ " in Portuguese sounds like the French " $j$ " instead of like the Spanish aspirate "g" ${ }^{16}$

O eufemismo que evita a menção à ruptura constitucional representada pela forma da tomada de poder de Vargas - "a new president about to take Office in Brazil" - é interessante, mas muito mais interessante é a idéia que a matéria sugere de que New York discutia eventuais pronúncias do nome do político - "considerable interest was expressed yesterday in New York as to the correct pronunciation of his name" - e que o Consulado Geral teria sido chamado a dirimir a questão: "Inquiry at the Brazilian Consulate General brought the explanation". 
A comparação fonética de sons do português brasileiro com o francês e com o espanhol parece reduzir todo o mundo não anglófono ao mesmo patamar de curiosidade e de exotismo, no que exotismo e curiosidade poderiam manifestar-se na pronúncia de certas letras: a fonética inglesa torna-se o padrão. Este auto-centramento da cultura norte-americana descortinado num breve passeio pelos jornais e correspondência permite, talvez, qualificar melhor, problematizando, a ironia e o ceticismo com que o escritor pensa o Brasil desde Nova Iorque. Já antes de 1927 ele se afastava do coro ufanista a partir da criação da polêmica figura do Jeca que tanto chocou patriotas. Mas é depois de 30 que sua crítica torna-se mais violenta.

E não há - e nem deve haver - como apaziguar contradições.

As idéias de Monteiro Lobato sobre o Brasil e sobre os Estados Unidos mudam ao longo de sua vida. A sociedade norte-americana que tantos elogios the inspira quando ele lá desembarca, ganha outra análise na sua correspondência dos anos quarenta, quando o escritor, de Buenos Aires, põe no mesmo saco o Estado Novo de Vargas e o anti-comunismo de Truman, como se vê em algumas cartas até agora inéditas, dirigidas a uma jovem professora gaúcha, Dona Therezinha Antunes:

\section{$15,11,47$}

Prezada amiga Tereza: ... A perseguição do Truman \& Cia contra os que não pensam como eles, corresponde a um enterro da batata. Se a batata está à flor da terra, vista por todos, analisada e criticada por todos, e correndo toda sorte de riscos ( ser pisada por um casco, ou devorada por um porco ), a batata terá pouca probabilidade de sobreviver e vencer. Mas se a enterram, ah, tudo muda. Ela pode germinar e brotar lindamente.

O que Truman e o resto da tropa estão fazendo com o comunismo, é apenasmente isso: enterrando a batata. E se não é precisamente o que o comunismo quer é 
indubitavelmente o de que ele precisa. O que ele precisa para vencer. O que aqueles imperadores romanos, sobretudo Domiciano, fizeram com o comunismo da época, vulgo cristianismo, foi enterrar a batata. E tão bem enterrada ficou ela depois de Domiciano, que explodiu no reinado de seu sucessor Constantino numa pujantíssima vegetação vitoriosa. Inda hoje li um rodapé de Tristão de Ataíde na Folha da Manhã, em que ele, católico e anti-comunista que é, lamenta a cruzada anti-comunista empreendida, a qual outra coisa não fará senão fomentar e "idealizar" o comunismo com os nimbos do martírio. ${ }^{17}$

\section{B.A. 8.4.47.}

Terezinha :... A bomba atômica é um perigo enquanto estiver com um só - e a linguagem de Truman passará a ser muito outra quando o seu país correr o risco de sofrer o que o Japão sofreu. Ora, a Rússia deve estar adiantadíssima nos estudos atômicos; e como eles sabem guardar segredo, é até de crer que estejam muitosíssimos mais adiantados do que pensamos . A atitude precipitada de Truman indica isso. É como quem diz: "Vamos aproveitar quanto antes da nossa superioridade, enquanto a temos, porque depois ... "Esperemos na Rússia, minha amiga ... ${ }^{18}$

Esperar na Rússia - conselho do velho Lobato (em 1947) a uma jovem brasileira documenta o namoro de Monteiro Lobato com o comunismo, flirt muito breve, na esteira de um longo e sólido namoro com o American way of life. A contradição é sugestiva: aponta para a extrema mobilidade de Monteiro Lobato ao longo do arco de opções ideológicas e políticas disponíveis para os intelectuais brasileiros de seu tempo. A contradição encontra também registro em várias linguagens e em vários gêneros, como trabalhos mais contemporâneos 
estão mostrando. 19 Manifesta-se, por exemplo, numa frase pinçada da carta, de fevereiro de 1929, enviada para Alarico Silveira, em que uma paisagem invernal nova-iorquina é lindamente tropicalizada: Aos olhos de Lobato, "A neve chegou e cobriu tudo com o seu manto de coco ralado" (Lobato, Cartas 118).... imagem bela e sugestiva de contradições e de dialéticas e, com a qual se encerram estas maltraçadas ...

\section{Notas}

* Prof. Lajolo has just received the prestigious Jabuti prize for "Monteiro Lobato (1882-1948) e outros modernismos," editors Marisa Lajolo and Luis Ceccantini (São Paulo: Editora UNESP, 2008); it is actually a double Jabuti for best publication in Theory/Literary Criticism, and best non-fiction publication of the year.

1. Agradeço o apoio do CNPq e da FAPESP à pesquisa que tornou possível o presente trabalho. Igualmente, a pesquisa no banco de dados do NYT, mencionada neste trabalho contou com o inestimável apoio de Dartmouth College. Ficam registrados os agradecimentos aos professores Rodolfo Franconi e Raul Bueno Chavez pelo convite. E a Miguel Valladares, sem cujo auxílio os bancos de dados aqui mencionados continuariam indecifráveis.

2. New York Times (1857- Current File) Aug 17, 1927, ProQuest Historical Newspapers. The New York Times, p. 40.

3. Consulado Geral em Nova York em 20/ 21/ 11/ 30. NP. Boato assassínio S. Sampaio. 136

4. Embaixada em Washington em 26/27/5/27 95 .

5. Exteriores (Telegrama para BRASEMB em 28/5/27.49 - resposta 95 )

6. A revista Time ocupa-se do assunto: "Foreign News Petals Over Olga Jun. 10, 1929 B -BRAZIL Biggest news of the week to Rio de Janeiro editors was neither politics, crime nor disaster, but the arrival in the U. S. of Miss Olga Bergamini De Sa, "Miss Brazil," for an international beauty contest to be held June 8-12 in Galveston, Tex. shouldering other matter from Rio's front pages were rapt descriptions of how Manhattan welcomed shapely Olga. Rio editors dissertated on the significance of the occasion. The Correio de Manh said: "We recently had the 
honor of entertaining President Hoover, whom we applauded as representing the intelligence, culture and merit of the great American nation. Now we reciprocate by this visit."'"Olga not only represents the feminine beauty of our race but the charm of the Brazilian woman.... Each petal unfolding over Olga's young crowned head is for us like the stars sparkling in our flag." The more conservative O Jornal said: "Miss Brazil's mission has grown to proportions that none expected.... Her smile brings the two republics closer than arduous diplomacy. We Brazilians are grateful to the Americans for the distinctions shown to our countrywomen. Public opinion is grateful to the great sister republic of the North which each day becomes more beloved by Brazilians." Whether the Brazilian editors knew it or not, Miss Brazil was but one of many Manhattan arrivals from far lands for the Galveston contest. Her presence, like theirs, received nothing more than routine mention, even in the tabloid press where stories and pictures of female pulchritude are so standardized that it is scarcely necessary to change the names from day to day. Characteristic was an item in Variety, theatre weekly, which published an article on the hotel accommodations and diet of the Galveston contestants, entitled FOREIGN BEAUTS CRAVE HOT MEAT." Fonte: TIME Magazine. Disponível em: http:/ / www.time.com/time/archive/preview/0,10987,751933,00.html Acesso em 09 fev. 2005.

\section{Cf. Cedae MLb 3.2.00407cx8}

8. Cf. Herder. Lá a coleção é definida como uma " series of (...) small (31/2 x 5 inch) paper covered booklets published in Girard (Kansas) [...] printed in standardized lengths of 32, 64, 96 and 128 pages,. (...) the Little Blue Books were explicitly conceived of 'as agency of popular culture'" (881-891).

9. O original de Modern Torture, Suplício moderno é datado de 1916; o de The Penintent Wag, O engraçado arrependido é igualmente datado de 1916 e nota da edição de informa que o conto foi originalmente publicado na Revista do Brasil de 16.04.1917 com o título A gargalhada do Colector. O original de The Pantation Buyer, O comprador de fazendas também é datado de 1916.

10. SECRETARIA DE ESTADO DAS RELAÇÕES EXTERIORES. Em 19 de Agosto de 1927 No PX/ 17 Índice: O uso da língua portuguesa na União das Repúblicas Americanas.

11. SECRETARIA DE ESTADO DAS RELAÇÕES EXTERIORES Em 22 de Novembro de 1927 N PA/ 27. CONFIDENCIAL Índice: Movimento anti-americano na Conferência da Havana. 
12. Cf. Biblioteca Monteiro Lobato. Pasta 33_002 lvl \& 003lvl.

13. CONSULADO EM NOVA YORK EM 15/12/30. C. 314.162 Transferência de serviços para o Ministério do Trabalho 97.

14. Dados extraídos de carta enviada a Monteiro Lobato por Octales M.Ferreira em nome da Companhia Editora Nacional em 27 de fevereiro de 1941 (Cedae MLb 3.2.00407cx8).

15. NYT ( 1857-Current file) May 22, 1927. ProQuest Historical Newspapers. The New York Times.p. E1.

16. New York Times (1857- Current File) Oct. 28, 1930, ProQuest Historical Newspapers. The New York Times, p. 28.

17. Carta depositada no Cedae/IEL/Unicamp BL Ms00013

18. Carta depositada no Cedae/IEL/Unicamp BL Ms00011

19. Cf. Lajolo, Marisa e José Luis Ceccantini.

\section{Referências}

FORD, H.;CROWTHER H. My Life and Work. New York: Doubleday, Page \& Company, 1922. <http:// www.gutenberg.org/dirs/etextos/hnfrd10.txt>.

HERDER, DALE M.; HALDERMAN J. The Little Blue Books , and the Theory of Popular Culture. Journal of Popular Culture 4.4 (Spring 1971): 881- 891.

LAJOLO, M.; CECCATINI, J. Monteiro Lobato livro a livro (obra infantil). São Paulo: IMESP-EDUNESP, 2008.

LOBATO, M. A barca de Gleyre: correspondência de Monteiro Lobato com Godofredo Rangel. São Paulo: Editora Brasiliense, 1951. 2 vols. . Cartas escolhidas. São Paulo: Editora Brasiliense. 6a. ed. 1970. 
